\title{
A STUDY OF POSTERIOR FOSSA MALFORMATIONS: MR IMAGING
}

Ravi Ningappa ${ }^{1}$, Vaishali D. M², Vijayaraghavachari T. V³, Manjappa B. $\mathrm{H}^{4}$

\section{HOW TO CITE THIS ARTICLE:}

Ravi Ningappa, Vaishali D. M, Vijayaraghavachari T. V, Manjappa B. H. "A Study of Posterior Fossa Malformations: MR imaging". Journal of Evolution of Medical and Dental Sciences 2015; Vol. 4, Issue 14, February 16; Page: 2254-2262, DOI: 10.14260/jemds/2015/330

ABSTRACT: AIMS AND OBJECTIVES: The aim of our study is to describe the imaging findings of various posterior fossa malformations and to evaluate the supratentorial abnormalities associated with posterior fossa malformations. MATERIALS AND METHODS: MR images of 30 patients with posterior fossa malformations detected in the department of Radiodiagnosis, BMCRI over a period of two years, from December 2012 to December 2014 were evaluated retrospectively. The various posterior fossa malformations were evaluated. Associated supratentorial abnormalities were noted. RESULTS: 30 patients with posterior fossa malformations were included in the study. The age group of patients ranged from 1year to 53years. There were 18 males and 12 females. The various posterior fossa malformations detected were Dandy Walker malformation (1 case), Dandy Walker variant ( 2 cases), mega cisterna magna (8 cases), arachnoid cysts ( 5 cases), Chiari 1 malformation (5 cases), Chairi 2 malformation (2 cases), Joubert malformation (1 case), lipoma (2 cases), vermian and/or cerebellar hypoplasia without posterior fossa CSF collection or cyst (4 cases). Associated supratentorial abnormalities were seen in 8 cases. CONCLUSION: MRI is the imaging modality of choice in the evaluation of posterior fossa malformations. It is very important to know the imaging findings of these malformations and to have knowledge about the various supratentorial and spinal abnormalities associated with them so as to provide an accurate diagnosis which is very essential for predicting the prognosis and planning further management.

KEYWORDS: Uterine artery, uterine vein, varicose vein, hemoperitoneum, laparotomy.

INTRODUCTION: MRI due to its multiplanar capabilities and exquisite contrast differentiation is the imaging modality of choice in the evaluation of congenital anomalies of brain. Although few posterior fossa malformations are rare, few are commonly encountered in day to day practice and since, imaging plays an important role in the accurate diagnosis of these malformations, it is very important for every radiologist to be familiar with the basic imaging findings of these anomalies. The aim of our study is to describe the imaging findings of various posterior fossa malformations and to evaluate the supratentorial abnormalities associated with posterior fossa malformations.

MATERIALS AND METHODS: MR images of 30 patients with posterior fossa malformations detected in the department of Radiodiagnosis, BMCRI over a period of two years, from December 2012 to December 2014 were retrospectively analysed.

Images were obtained with a Siemens Vision 1.5T MR scanner. Sagittal T1, axial T1W, T2W and coronal T2W images were evaluated. The various posterior fossa and supratentorial structures were evaluated.

The cerebellar hemispheres and vermis were evaluated for hypoplasia, atrophy, rotation. Posterior fossa was considered enlarged if the tentorium, torcular and sinuses are elevated. Presence or absence of posterior fossa CSF collections, cysts was noted. Brainstem was evaluated for 
size, displacement and morphology. Ventricular system and supratentorial structures were also evaluated to look for associated abnormalities.

RESULTS: 30 patients with posterior fossa malformations were included in the study. The age group of patients ranged from 1 year to 53 years. There were 18 males and 12 females. The various posterior fossa malformations detected were Dandy Walker malformation (DWM, 1 case), Dandy Walker variant (DWV, 2 cases), mega cisterna magna (MCM, 8 cases), arachnoid cysts (5 cases), Chiari 1 malformation (5 cases), Chairi 2 malformation (2 cases), Joubert malformation (1 case), lipoma ( 2 cases), vermian and/or cerebellar hypoplasia without posterior fossa cysts (4 cases). Most common posterior fossa malformation in our study was mega cisterna magna.

Supratentorial abnormalities were seen in 8 cases. The supratentorial findings in these cases were hydrocephalus, corpus callosum agenesis, agyria, cerebral atrophy, open lip schizencephaly and craniosynostosis.

Posterior fossa CSF collections communicating with fourth ventricle/ subarachnoid space were noted in 11 cases ( 1 case of DWM, 2 cases of DWV, and 8 cases of MCM). Out of this, one case with DWM had enlarged posterior fossa with elevated tenorium, torcular. Posterior fossa was normal in size in the other cases. Inferior vermian hypoplasia was noted in both cases of DWV. Vermis was normal in size in mega cisterna magna. Associated abnormalities included hydrocephalus in DWM, corpus callosum agenesis with left inferior cerebellar hypoplasia in 1 case of DWV, and gross communicating hydrocephalus in one case of mega cisterna magna.

Posterior fossa cyst with no definite communication with fourth ventricle was noted in all 5 cases of arachnoid cyst.

Vermian hypoplasia with or without cerebellar hypoplasia, without posterior fossa cyst or enlargement was noted in 4 cases. All the 4 cases had vermian hypoplasia. Two cases had associated supratentorial abnormalities - open lip schizencephaly with corpus callosum agenesis (1 case), cransynostosis, agyria with bilateral cerebral and cerebellar atrophy, ventriculomegaly (1 case).

Chiari malformations constituted 7 cases. Small posterior fossa was noted in 2 cases of Chiari 2 malformation with descent of posterior fossa structures through foramen of magnum. Associated abnormalities were hydrocephalus in both the cases of Chiari 2 malformation, upper cervical myelomeningocele in one case and lumbar myelomeningocele in other case. All the 5 cases of Chiari 1 malformation had tonsillar ectopia of $>5 \mathrm{~mm}$. Hydrosyringomyelia was noted in 3 cases of Chiari 1 malformation and obstructive hydrocephalus in one case.

DISCUSSION: Posterior cranial fossa is the largest cranial fossa. It contains brainstem (Pons, medulla) anteriorly and vermis and cerebellar hemispheres posterolaterally. The midbrain lies within the tentorial incisura and represents the transition between the cerebral hemispheres and hindbrain. CSF containing spaces of posterior fossa include cerebral aqueduct, fourth ventricle and CSF cisterns around brainstem and cerebellum.

\section{Posterior fossa malformations Include1:}

1. Cystic posterior fossa malformations - Dandy Walker malformation, Dandy Walker variant, mega cisterna magna, Blake's pouch cyst, arachnoid cyst.

2. Chiari malformations - Chiari 1 to IV and Chiari variants. 


\section{ORIGINAL ARTICLE}

3. Miscellaneous malformations - cerebellar hypoplasia, pontine tegmental cap dysplasia, rhombencephalosynapsis, Joubert syndrome, lipoma, epidermoid cysts.

I. Cystic posterior fossa Malformations: These include Dandy Walker malformation, Dandy Walker variant, mega cistern magna and posterior fossa arachnoid cyst.

Dandy Walker Malformation: Dandy Walker malformation is the cystic malformation of posterior fossa resulting from a defect in the area membranacea superior and is characterized by triad of cerebellar digenesis, cystic dilation of fourth ventricle and large posterior fossa with superior displacement of the tentorium, torcular and dural sinuses. ${ }^{1}$

Imaging findings incude large posterior fossa with high riding tentorium, torcular and straight sinuses, absent falx cerebeli, scalloping of inner table of occipital bone and petrous pyramids, dilated fourth ventricle communicating with a large posterior fossa cyst, absent or hypoplastic vermis, anterosuperior displacement of the remnant vermis, variable degrees of cerebellar hypoplasia (Figure 1). Supratentorial abnormalities associated with DWM include hydrocephalus, hypogenetic corpus callosum, grey matter heterotopias, aqueductal stenosis. ${ }^{2}$ Hydrocephalus was noted in one case of DWM in our study.

Dandy Walker Variant: The term Dandy Walker variant is now obsolete and is replaced by the term vermian-cerebellar hypoplasia. ${ }^{3}$ It is considered to be a milder form of Dandy Walker malformation. Imaging findings include normal sized posterior fossa, keyhole configuration of fourth ventricle communicating with a prominent cistern magna through a widely patent vallecula, varying degrees of vermian hypoplasia (Figure 2). ${ }^{1}$ Corpus callosum agenesis with left inferior cerebellar hypoplasia was noted in one case of DWV in our study.

Mega cistern Magna: Mega cisterna magna represents the mildest end of Dandy Walker spectrum. It is characterised by a large posterior fossa with an enlarged cisterna magna with normal fourth ventricle, vermis, and supratentorial brain (figure 3 ). ${ }^{1}$ The occipital bone may be scalloped. Cisterna magna is crossed by veins and falx cerebelli and it communicates with the fourth ventricle and subarachnoid space. ${ }^{4}$

Posterior fossa arachnoid cyst: Arachnoid cysts are benign developmental cavities of subarachnoid space containing fluid similar in composition to that of CSF and lined by membrane of true arachnoid cells. Most arachnoid cysts are suprasellar. Retrocerebellar and lateral cerebellopontine angle are the most common infratentorial locations. On imaging arachnoid cysts are extra axial cysts that follow CSF on all sequences and exert mass effect on adjacent brain and bone. ${ }^{5}$ Arachnoid cysts do not communicate with the fourth ventricle and veins and falx cerebelli do not cross the cyst. ${ }^{1}$

II. Chiari Malformations: Three malformations associated with hydrocephalus were described by Chiari in $1891 .^{6} \mathrm{~A}$ fourth one was described by him five years later. ${ }^{7}$ Recently Chiari variants have been described. 
Chiari I Malformation: Chiari I malformation is characterized by inferior displacement of cerebellar tonsils into the rostral cervical spinal canal (Figure 4). It presents in the second or third decade of life. Symptoms include suboccipital headache, ocular disturbances, cranial neuropathies, sleep apnea, and spinal cord disturbances. ${ }^{8}$

Degree of tonsillar ectopia has been considered the most important criterion for the diagnosis of Chiari I malformation, tonsillar ectopia of $>6 \mathrm{~mm}$ (Below the nasion - opisthion line) between the ages of 5 and 15 years and tonsillar ectopia of $>5 \mathrm{~mm}$ outside the 5-15 year age group is considered pathologic and is associated with increased incidence of clinical symptoms. Also, symptomatic Chiari I malformations are associated with a pointed configuration of the cerebellar tonsils. ${ }^{9}$ All the cases of Chiari 1 malformation in our study had tonsillar ectopia of $>6 \mathrm{~mm}$. Three cases had associated hydrosyringomyelia of cervical and/or thoracic spinal cord and one case had obstructive hydrocephalus likely secondary to aqueductal stenosis.

Other imaging findings include compression of the cerebellar cisterns, reduced height of the supraocciput, short clivus, anterior displacement of the cerebellum, kinking of the medulla, retroflexion of odontoid process. ${ }^{8}$

Associated abnormalities include syringohydromyelia in 25\%-60\% patients, craniovertebral junction abnormalities, and hydrocephalus. ${ }^{9}$

Chiari II Malformations: Chiari II malformation involves displacement of the brainstem and lower cerebellum into cervical spinal canal (figure 5). Almost all are associated with lumbar myelomeningoceles. 5 Usually present at birth due to lumbar myelomeningocele. Symptoms include brainstem dysfunction, cranial nerve palsies, neck pain, and sleep apnea.

According to Mclone and Knepper, ${ }^{10}$ abnormal neurulation is the primary defect in Chiari II malformation that results in abnormal leakage of CSF leading to disruption of the normal ventricular enlargement which in turn leads to the various structural abnormalities seen in Chiari II malformations.

Bony and dural abnormalities include small, shallow bony posterior fossa, large, gaping foramen magnum, concave petrous temporal bones, short concave clivus, widened heart shaped tentorial incisura, fenestrated falx, irregular interhemispheric fissure. ${ }^{1}$

Brainstem and cerebellar abnormalities include inferior displacement of the medulla and cerebellar vermis into the upper cervical spinal canal, cervicomedullary kink with a medullary spur, beaked tectum, anteromedial displacement of the cerebellar hemispheres around the brainstem, towering cerebellum due to superior displacement of the cerebellum and vermis through the incisura. ${ }^{1}$

Ventricular abnormalities include thin and elongated fourth ventricle that lacks a fastigium, large massa intermedia, hydrocephalus. ${ }^{1}$

Most of the patients with Chiari II malformation have supratentorial abnormalities that include malformations of cortical development, callosal dysgenesis, enlarged caudate heads, stenogyria, fenestrated falx. Spinal cord abnormalities include lumbar myelomeningocele (in almost all cases), hydrosyringomyelia (50\% cases). ${ }^{1,7}$

\section{Miscellaneous posterior fossa Malformations:}

Joubert malformation: Joubert malformation is the term used for vermian hypogenesis with separation of cerebellar hemispheres and Joubert's clinical syndrome. ${ }^{11}$ Signs and symptoms include hypotonia, developmental delay and impaired equilibrium. ${ }^{12}$ Imaging findings include deepening of 


\section{ORIGINAL ARTICLE}

the interpeduncular fossa, elongation and thinning of isthmus, thick abnormally oriented superior peduncles (Figure 6). The appearance of mesencephalon and superior cerebellar peduncles has been described as molar tooth sign. There is variable degree of vermian hypoplasia with an abnormal vermian cleft which runs anteroposteriorly. Varying degrees of fourth ventricular enlargement is noted with bat shaped configuration of rostral fourth ventricle. ${ }^{13}$

Cerebellar Hypoplasia: Cerebellar hypoplasias show a spectrum of findings with small cerebellar hemispheres and vermis (Figure 7). In severe cases, cerebellar hemspheres and vermis are totally absent with an empty posterior fossa. ${ }^{1}$

Lipoma: Intracranial lipomas are considered disorders of development of subarachnoid space and are believed to arise from mal differentiation of meninx primitive. ${ }^{5}$ They are often asymptomatic and are found incidentally. The common sites of intracranial lipomas in the decreasing order of frequency include pericallosal region, quadrigeminal/superior cerebellar cistern, suprasellar and cerebellopontine angle cisterns. ${ }^{14}$ Lipomas are frequently associated with congenital abnormalities of adjacent neural tissue in $60 \%$ cases. On MRI, intracranial lipomas exhibit signal characteristics similar to fat, they appear hyperintense on T1 weighted image, intermediate signal on T2 weighted image and are suppressed on fat-suppressed image (Figure 8).

CONCLUSION: We have described the MR imaging findings of some of the posterior fossa malformations and associated abnormalities. We hereby conclude that, MRI is the imaging modality of choice in the evaluation of posterior fossa malformations. It is very important to know the imaging findings of these malformations and to have knowledge about the various supratentorial and spinal abnormalities associated with them so as to provide an accurate diagnosis which is very essential for predicting the prognosis and planning further management.

\section{REFERENCES:}

1. Posterior fossa malformations. In: Osborn AG. Osborn's Brain: Imaging, Pathology, and Anatomy, 1st Edition. Amirsys Publishing, Inc, 2013: 1055-1081.

2. Altman N, Naidich T, Braffman B. Posterior fossa malformations. AJNR Am J Neuroradiol 1992; 13: 691-724.

3. Kollias S, Ball W, Prenger E. Cystic malformations of the posterior fossa: differential diagnosis clarified through embryologic analysis. Radiographics 1993; 13: 1211-1231.

4. Tortori-Donati P, Fondelli M, Rossi A, et al. Cystic malformations of the posterior cranial fossa originating from a defect of the posterior membranous area. Child's Nerv Syst 1995; 12: 303308.

5. Caruso PA, Robertson R, Setty B, Grant E. Disorders of brain development. In: Scott W. Atlas. Magentic resonance imaging of the brain, $4^{\text {th }}$ edition. Lipincott Williams and Wilkins, 2009: 194-270.

6. Chiari H, Über Veränderungen des Kleinhirns infolge von Hydrocephalie des Grosshirns. Deutsch Medizinische Wocherschrift1891; 17: 1172-5. 


\section{ORIGINAL ARTICLE}

7. Chiari H. Über Veränderungen des Kleinhirns, des Pons unde der Medulla Oblongata infolge von congenitaler Hydrocephalie des Grosshirns. Denkschrift Kais Akad Wiss Math-Naturw 1896; 63: 71-116.

8. Milhorat T, Chou M, Trinidad E, et al. Chiari I malformation redefined: clinical and radiographic findings for 364 symptomatic patients. Neurosurgery 1999; 44: 1005-1017.

9. Barkovich A, Wippold F, Sherman J, et al. Significance of cerebellar tonsillar ectopia on MR. AJNR Am J Neuroradiol 1986; 7: 795-799.

10. (McClone D, Knepper P. The cause of Chiari II malformation: a unified theory. Pediatr Neurosci 1989; 15: 1-12).

11. Shaw C-M, Alvord EJ. Hydrocephalus. In: Duckett S, ed. Pediatric neuropathology. Malvern, PA: Williams \& Wilkins, 1995: 152-159.

12. Maria B, Bolthauser E, Palmer B, et al. Clinical features and revised diagnostic criteria in Joubert syndrome. J Child Neurol 1999; 14: 583-590.

13. Quisling R, Barkovich AJ, Maria B. Magnetic resonance imaging features and classification of central nervous system malformations in Joubert syndrome. J Child Neurol 1999; 14: 628-635.

14. Truwit C, Barkovich A. Pathogenesis of intracranial lipoma: an MR study in 42 patients. AJNR Am J Neuroradiol 1990; 11: 665-674.

Figure 1: Dandy Walker malformation. A. Sagittal T1W MR image of brain shows large posterior fossa with hypo plastic, anterosuperiorly rotated vermis (white arrow) and elevated tentorium and dural sinuses (white arrow head). B. Axial T1W MR image of brain at the level of fourth ventricle showing cystically dilated fourth ventricle communicating with a large posterior fossa cyst. The patient also had gross hydrocephalus and thinned out corpus callosum.

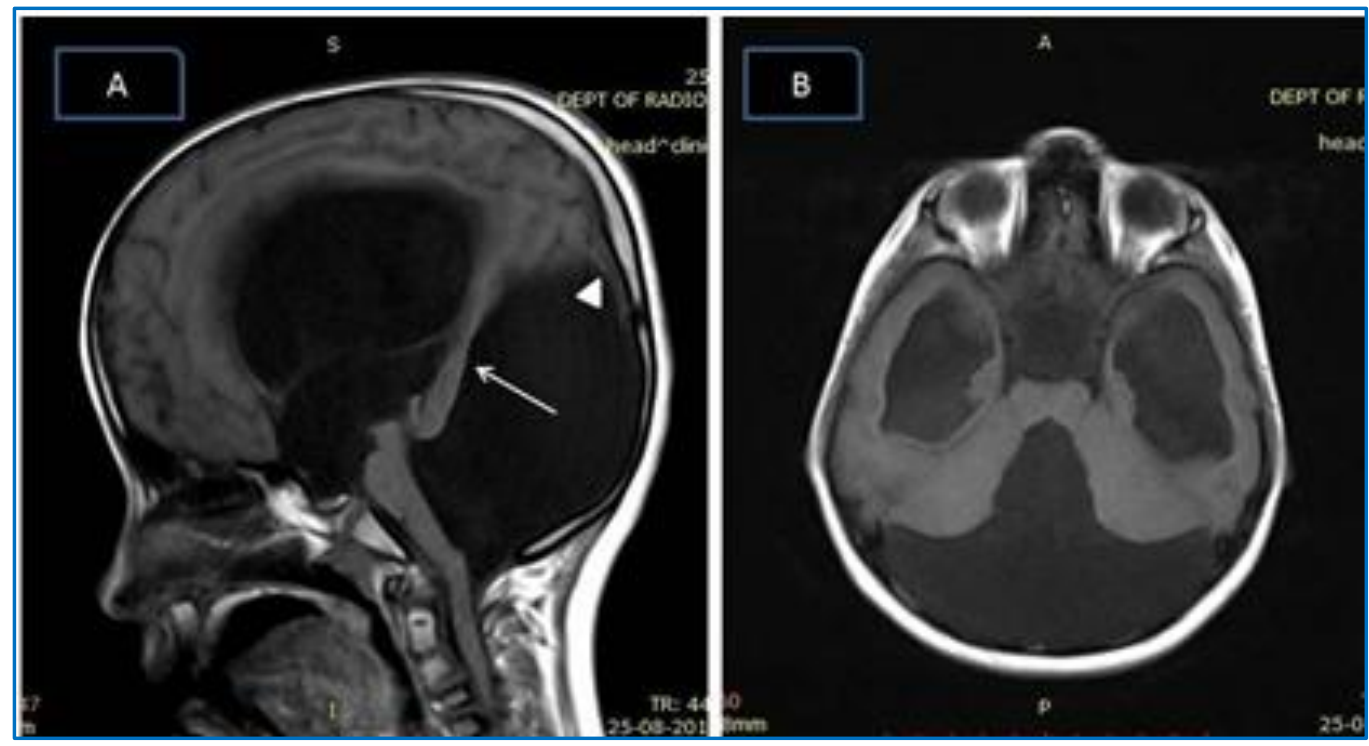

Fig. 1 


\section{ORIGINAL ARTICLE}

Figure 2: Dandy Walker Variant. A. Axial T1W MR image of brain showing key hole configuration of fourth ventricle (black arrow) communicating with a prominent cistern magna (White arrow). B. Sagittal T1W MR image of the same patient as in A, showing hypoplastic inferior vermis (white arrow).

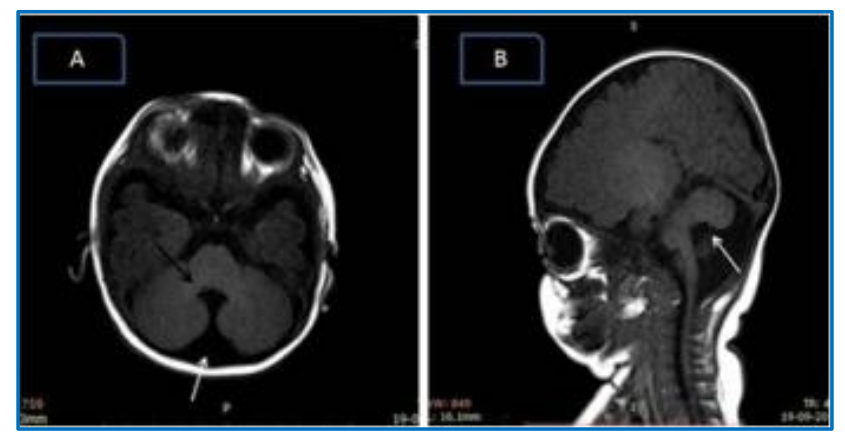

Fig. 2

Figure 3: Mega cisterna magna with cavum vergae. A. Axial T1W MR image shows a mega cistern magna. B. Axial T1W Mr image in the same patient at a higher level shows a cavum vergae (anatomical variant).

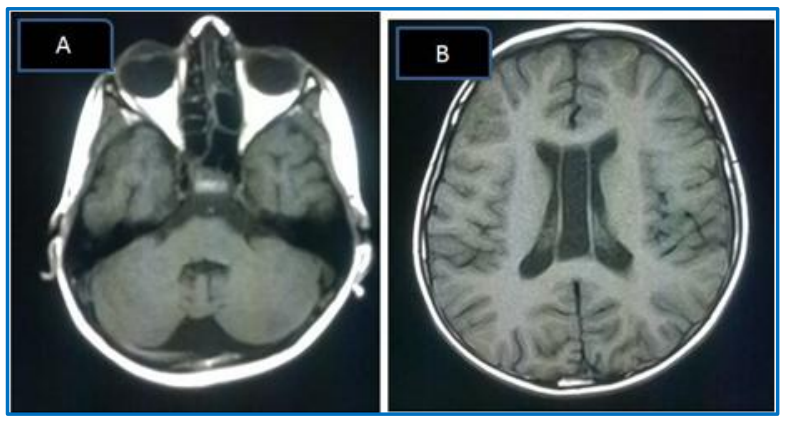

Fig. 3

Figure 4: Chiari 1 malformation. A. Sagittal T1W MR image of the brain and upper cervical spine shows inferior displacement of the cerebellar tonsils into the upper part of cervical spine (black arrow), and hydrosyringomyelic cavity in lower cervical spinal cord (white arrow). B. Sagital T2W MR image in another patient showing tonsilar ectopia (white arrow head) and hydrosyringomyelia of lower cervical spinal cord (white arrow).

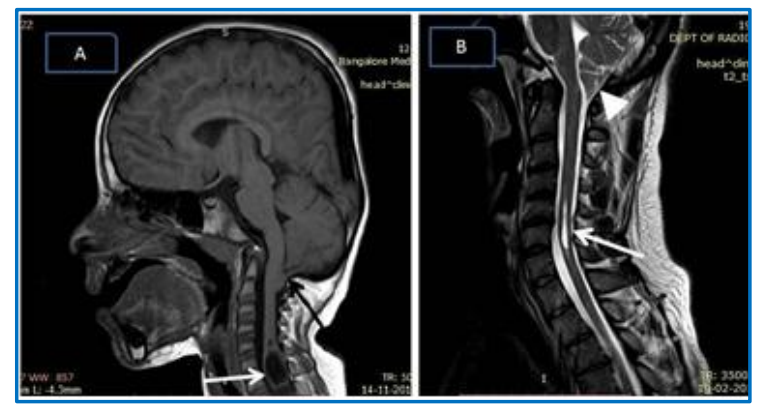

Fig. 4 


\section{ORIGINAL ARTICLE}

Figure 5: Chiari II malformation with cervical myelomeningocele. Sagittal T1W MR image of brain shows a small posterior fossa with a low positioned torcular. There is descent of posterior fossa structures through a widened foramen magnum (white arrowheads), with an effaced fourth ventricle (black arrow) and beaked tectum (short white arrow). The clivus is short (long white arrow) and there is moderate supratentorial hydrocephalus. Cervical myelomeningocele is present (black arrowhead).

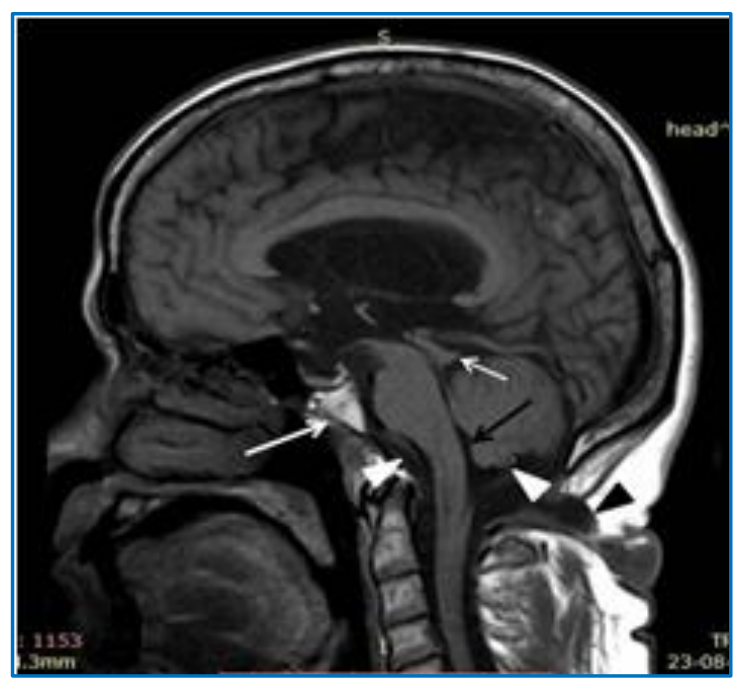

Fig. 5

Figure 6: Joubert anomaly. A. Axial T1W MR image of brain show elongated superior cerebellar peduncles (white arrow), prominent fourth ventricle and apposing cerebellar hemispheres (black arrow) secondary to vermian agenesis. B. Axial T1W images at the level of midpons showing bat wing appearance of fourth ventricle (white arrow) and apposing cerebellar hemispheres (black arrow).

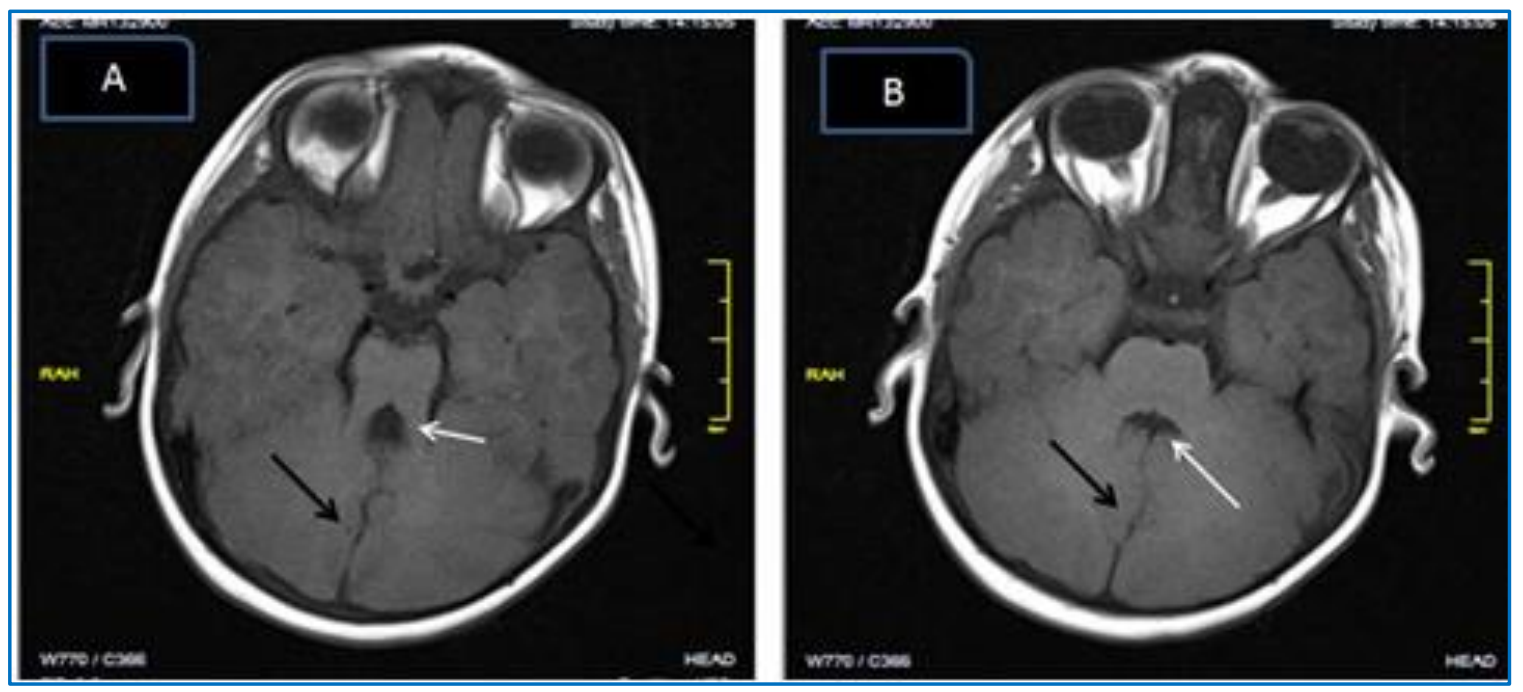

Fig. 6 


\section{ORIGINAL ARTICLE}

Figure 7: Bilateral cerebellar and inferior vermian hypoplasia associated with craniosynostosis, agyria and cerebral atrophy.

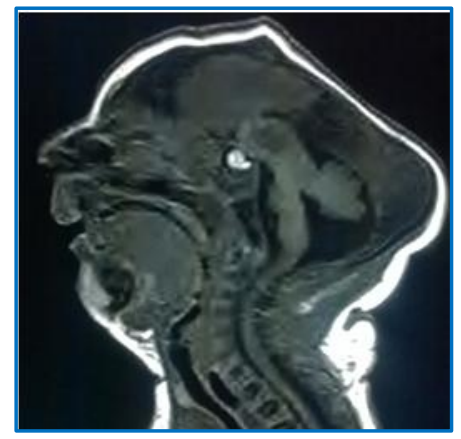

Fig. 7

Figure 8: Quadrigeminal cistern lipoma. A Well-defined $\mathrm{T} 1$ hyperintensity lesion in the quadrgeminal cistern (black arrowhead). Signal intensity of the lesion is similar to subcutaneous fat.

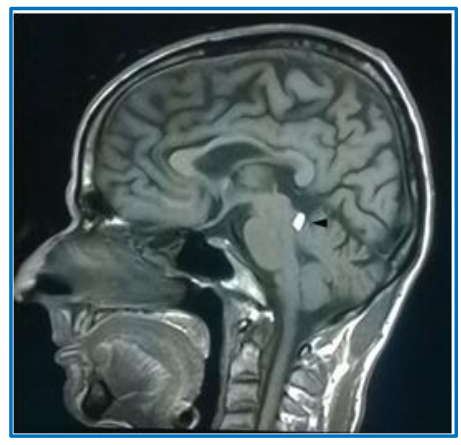

Fig. 8

\section{AUTHORS:}

1. Ravi Ningappa

2. Vaishali D. M.

3. Vijayaraghavachari T. V.

4. Manjappa B. H.

\section{PARTICULARS OF CONTRIBUTORS:}

1. Associate Professor, Department of Radiodiagnosis, BMCRI, Bangalore.

2. Resident, Department of Radiodiagnosis, BMCRI, Bangalore.

3. Resident, Department of Radiodiagnosis, BMCRI, Bangalore.

FINANCIAL OR OTHER

COMPETING INTERESTS: None
4. Resident, Department of Radiodiagnosis, BMCRI, Bangalore.

\section{NAME ADDRESS EMAIL ID OF THE} CORRESPONDING AUTHOR:

Dr. Ravi Ningappa,

Associate Professor,

Department of Radiodiagnosis,

Bangalore Medical College and

Research Institute, Bangalore.

E-mail:raviningappa6@gmail.com

Date of Submission: 04/01/2015.

Date of Peer Review: 05/01/2015.

Date of Acceptance: 07/02/2015.

Date of Publishing: 13/02/2015. 